\title{
Arthroscopic release of iliopsoas tendon in patients with femoro-acetabular impingement: clinical results at mid-term follow-up
}

\author{
Rodrigo Mardones \\ Alessio Giai Via \\ Alexander Tomic \\ Claudio Rodriguez \\ Matias Salineros \\ Marcelo Somarriva
}

Department of Adult Reconstruction Surgery

Hip/Knee and Hip Arthroscopy,

Clínica Las Condes, Las Condes, Santiago de Chile, Chile

Corresponding author:

Rodrigo Mardones

Head of Department of Adult Reconstruction Surgery

Hip/Knee and Hip Arthroscopy, Clínica Las Condes

La Fontecilla 441, Las Condes, Santiago de Chile,

Chile

E-mail: rmardones@clc.cl

\section{Summary}

Background: The iliopsoas tendon is a recognized cause of extra-articular hip pain, and tenotomy has been described as an effective treatment in patients who do not respond to conservative treatments. Endoscopic release showed higher success rate, lower recurrence, fewer complications compared to open surgery. The aim of the study is to report the results at a mean of 4 years follow-up of a series of patients affected by femoroacetabular impingement (FAI) and an associated iliopsoas tendinopathy, treated with hip arthroscopy and transcapsular tendon release.

Methods: Fifteen patients were retrospectively reviewed. Assessment of radiographic signs of FAl was performed, the alpha angle, the femoral headneck offset and the lateral center edge angle (LCEA) were collected. Osteoarthritis was assessed from the AP pelvic and graded according to the Tönnis classification. Modified Harris Hip Score (mHHS), VAIL score and VAS score were administered to all patients before surgery, at follow-up at 1 year (T1) and final follow-up (T2).

Results: We found a statistical significant improvement in functional scores (mHHS and VAIL score) from the baseline to T2. According to VAS score, a statistical significant improvement was also found from T0 to T2, from a median of 5.5 (range 3-7) to 0 (range $0-5)(P<0.001)$. Two patients referred a recurrence of pain one year after surgery who were treated conservatively. No other complications have been reported.

Conclusion: Iliopsoas tendinopathy can be associated to FAl in some patients, and failure in diagnosing and treating may be the reason of poor results and a revision surgery. Arthroscopic iliopsoas tendon release seems to produce good clinical outcome, reducing pain and the rate of a revision surgeries. Level of evidence: IV case series.

KEY WORDS: hip arthroscopy, femoroacetabular impingement, iliopsoas tendon, tendinopathy, arthroscopic tendon release.

\section{Introduction}

Since hip arthroscopy was described in 1931 by Burman $^{1}$, and the first arthroscopic description of a labral tear was described by Suzuki et al. in $1986^{2}$, many progresses have been made in diagnosis and treatment of hip pathologies. Recent advances in diagnostic imaging and arthroscopic techniques allowed hip arthroscopy to improve, and many intra and extra-articular pathologies can be currently treated satisfactorily ${ }^{3}$. Good to excellent results have been reported after arthroscopic treatment of femoroacetabular impingement (FAI) at long term follow-up 4 .

The iliopsoas tendon has been gaining increased interest for its role as a generator of hip pain and its association with intra-articular pathologies, because of its the complex anatomic relationship with the anterosuperior labrum and the hip joint capsule. Intra-articular pathologies may be associated with iliopsoas tendinopathy up to $50 \%$ of cases, while iliopsoas tendon may produce a labral injury itself 5 .

Tenotomy has been described as an effective treatment in patients with iliopsoas tendinopathy or internal snapping hip syndrome which do not respond to conservative treatments ${ }^{6}$. Both open and arthroscopic surgical techniques are reported for iliopsoas tendon release with good results. However, a higher success rate, less recurrence, fewer complications, decreased postoperative pain and better cosmetics have been reported with endoscopic management compared to open surgery ${ }^{7}$. Failure to recognize the iliopsoas pathology while performing hip arthroscopy have been proved to be a cause of persistent pain and 
poor long-term outcomes ${ }^{8}$. For this reason, many Authors actually underline the importance of managing iliopsoas tendon disorders while performing intra-articular procedure 7,9

The aim of this study is to report the clinical and functional results at a mean of 4 years follow-up of a series of patients affected by FAI and an associated iliopsoas tendinopathy, treated with hip arthroscopy and transcapsular tendon release. The hypothesis is that arthroscopic FAI treatment and iliopsoas release reduce symptoms and improve hip function.

\section{Materials and methods}

We reviewed retrospectively the data on 15 patients (4 men, 11 women) who underwent hip arthroscopy and transcapsular iliopsoas tendon release for FAI with associated iliopsoas tendinopathy. All the patients have been treated in the period from May 2011 to December 2012. Both surgical and study procedures were performed after the patients had signed a written consent and after approval by the local Internal Review Board (IRB-Clínica Las Condes, Santiago de Chile, Chile). The study has been performed according the basic principles and recommendations in clinical and field science research ${ }^{10}$.

\section{Inclusion and exclusion criteria}

Inclusion criteria were clinical and imaging evidence of FAI and iliopsoas tendinopathy, patient older than 18 years and younger than 50 . Exclusion criteria were patient with X-ray evidence of hip osteoarthrosis (Tönnis grade 2 or higher), revision hip arthroscopy, patients with co-morbidities predisposing to osteoarthritis such as rheumatological disorders, avascular necrosis, Legg-Calvé-Perthes disease, EhlersDanlos syndrome, chronic renal failure, previous intra-articular fractures. Patients with total hip arthroplasty were also excluded.

\section{Patient assessment}

A detailed physical examination was conducted in all patients, who were examined by an orthopaedic surgeon with a full training and great experience in hip and pelvic surgery (R.M.).

Anterior impingement test was considered positive if pain was elicited in forced flexion combined with internal rotation of the hip (FADIR test) ${ }^{11}$. The FABER test was evaluated in all cases ${ }^{11}$. When the patient referred pain at the iliopsoas tendon palpation or at the palpation at the hip flexion against resistance, it was considered positive for iliopsoas tendinopathy. Evaluation of internal snapping of the iliopsoas tendon was performed as the hip was brought from a flexed, abducted, and externally rotated position into extension with internal rotation ${ }^{11}$. All physical examinations were performed and documented in degrees in a clinical setting.

All patients took standard weight-bearing anteriorposterior radiographs of the pelvis and cross-table view of the hip (Figure 1). Assessment of radiographic signs of $\mathrm{FAl}$ was also performed.

Cam impingement was assessed by the measure-

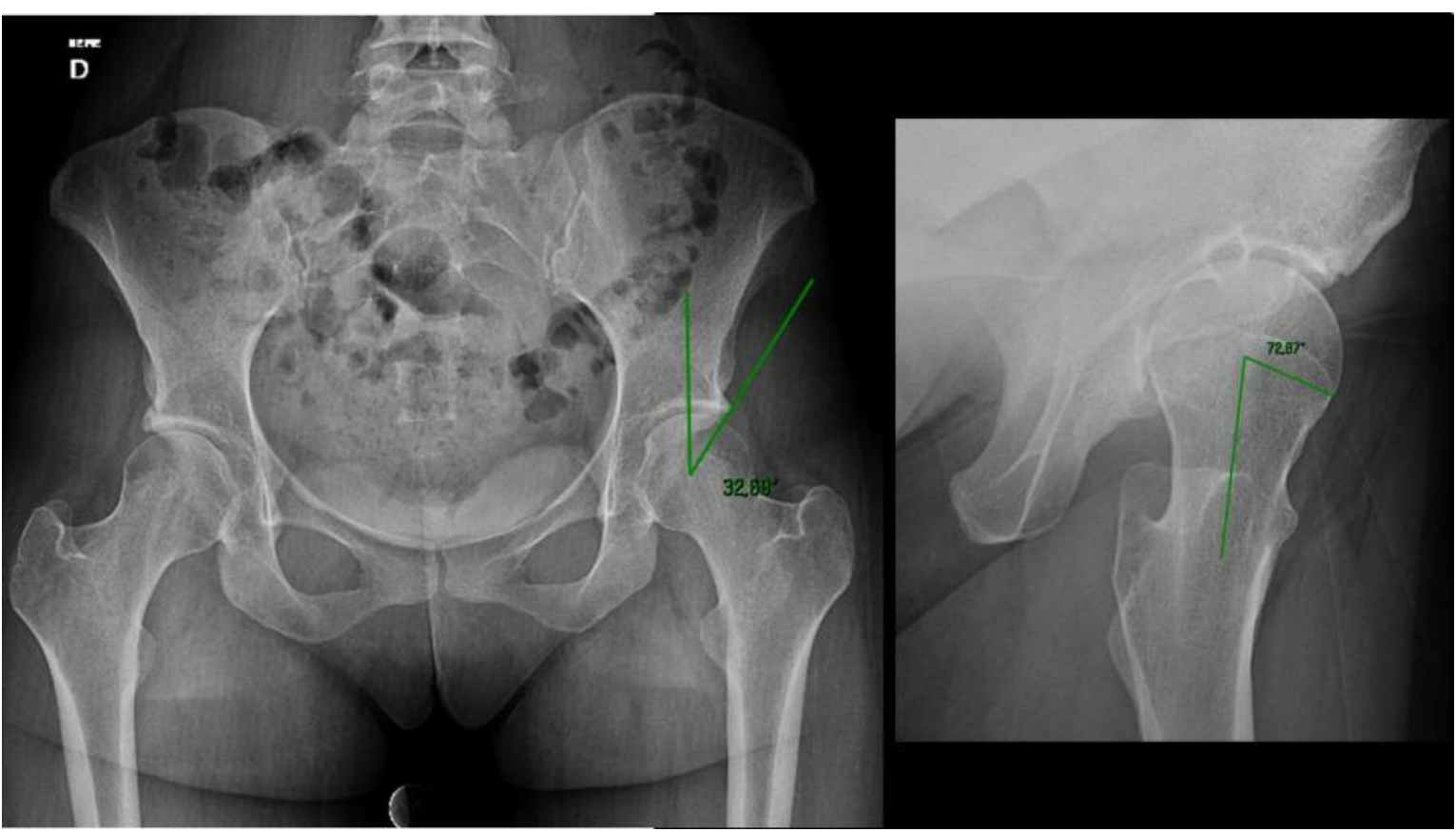

Figure 1. Preoperatory X-ray of a 22-year-old woman with a combined FAI and associated iliopsoas tendinopathy. The cross-over sign is detectable on the AP pelvis X-ray, and the bump with an $\alpha$-angle of $72^{\circ}$ is showed into the cross-table view of the hip. 
ment of the alpha angle on the cross-table view of the hip and femoral head-neck offset ${ }^{12}$. An alpha angle of greater than $50^{\circ}$ and a femoral head-neck offset lower than $10 \mathrm{~mm}$ were considered pathologic ${ }^{13}$. Pincer impingement was defined as a lateral center edge angle (LCEA) by Wiberg greater than $35^{\circ}$ and/or a extrusion index smaller than $20 \%{ }^{14}$. Acetabular retroversion, indicated by crossover sign, was explored for on AP pelvic view. The symphysis-coccyx distance was respected (1 to $2 \mathrm{~cm}$ ) to avoid functional retroversion due to anterior pelvic tilt. Osteoarthritis was assessed from the AP pelvic X-ray and graded according to the Tönnis classification ${ }^{15}$.

An artro-MRI of the affected hip was performed to diagnose the labral injury, chondral defects or tendinopathy of the ligamentum teres. All patients underwent a diagnostic intra-articular lidocaine injection to confirm the diagnosis.

Modified Harris Hip Score and Vail score were administered to all patients before surgery (TO), at follow-up at 1 year (T1) and final follow-up (T2). The VAS score was also administered preoperatively and at each control. Follow-up data were obtained through office visits with the operating surgeon (RMP) and a phone calls from a fellow (AGV; CRM). The date of the office visits or of the phone call was used as the follow-up date.

\section{Surgical technique}

Under spinal anaesthesia and superficial sedation, the patient was positioned supine. First traction was applied to the affected lower limb and the joint was distracted. To confirmed hip joint distraction an image intensifier was used. Than traction was released and the hip and the lower limb were prepared and draped in the usual sterile fashion. Chlorhexidine was used to prepare the skin and a clear drape (3M Health Care, St Paul, Minnisota, USA) was used for the limb. All arthroscopies were performed by the same surgeon (R.M.P.) After distract the hip joint, the anterolateral portal was performed by introducing a fluoroscopy-guided spinal needle in the central compartment. A $70^{\circ}$ arthroscope was used. A second modified anteromedial portal was established under direct vision $^{16}$.

A diagnostic round was performed into the joint and all pathologies were registered. After capsulotomy, direct signs of iliopsoas tendinopathy were look for, as hyperemia, macroscopic degeneration of the tendon or a labral tear at 3 o'clock. Then, tenotomy was performed through the central compartment at the level of the labrum (Figure 2). After iliopsoas tendon release, acetabuloplasty was performed taking into account acetabular and femoral anatomy, intra-articular damage, and ligamentous laxity. The labrum was repaired with 1 to 4 bonny anchors (S\&N) and microfractures were performed at the labrum-cartilage junction when it was injured. Then, the traction was released and the bumpectomy was performed. The cheilectomy was performed under direct vision and the hip was moved from flexion to full extension and rotated to check for correct resection of the bump. In all cases PRP was positioned at the head-neck junc-

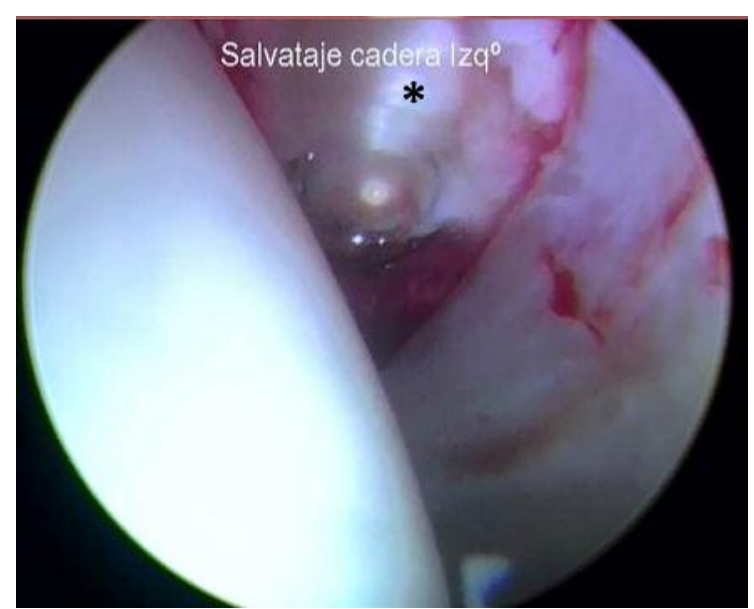

Figure 2. Intra-operative image of the transcapsular release of the iliopsoas tendon.

tion. The skin closed with subcuticular absorbable sutures. Post-operatively, all patients underwent supine standard X-ray of the pelvis and cross-table view of the hip (Figure 3).

\section{Post-operative rehabilitation}

Post-operative mobilization with crutches was initiated as soon as possible, and weight-bearing allowed as tolerated. Passive hip flexion-extension mobilization with Kinetec ${ }^{\circledR}$ and active mobilization was started immediately after surgery. Static bicycle was prescribed for 15 minutes twice a day from the first postoperative day. The patient started a rehabilitation program with a physiotherapist with expertise in hip surgery one week after surgery. At 4 weeks one crutch was removed, and full weight bearing without crutches is allowed 6 weeks after surgery. Celecoxib 200 mg twice a day was prescribed for 3 weeks as prophylaxis of heterotopic calcifications.

\section{Statistical analysis}

Descriptive statistics are presented as mean $( \pm S D)$. The T-Student test for independent samples was used to detect for differences between baseline and follow-up for each variable. A p value $<0.05$ was considered to be statistically significant. Statistical analysis was performed by using SigmaPlot 11.0 software (Systat Software, Tulsa, OK, USA).

\section{Results}

Nineteen patients (21 hips) were diagnosed for FAI and a transcapsular release of iliopsoas tendon. Of these, 1 patient was excluded because of a chodral injury, 1 was a revision hip arthroscopy and 2 patients were lost at follow-up. So, 15 patients (17 hips) who met the inclusion criteria were included into the study. The mean age was 33.5 years (range 18-49; S.D. 10.7). Patients referred hip pain from a mean of 12.3 month (min 3 months-max 48 months). 


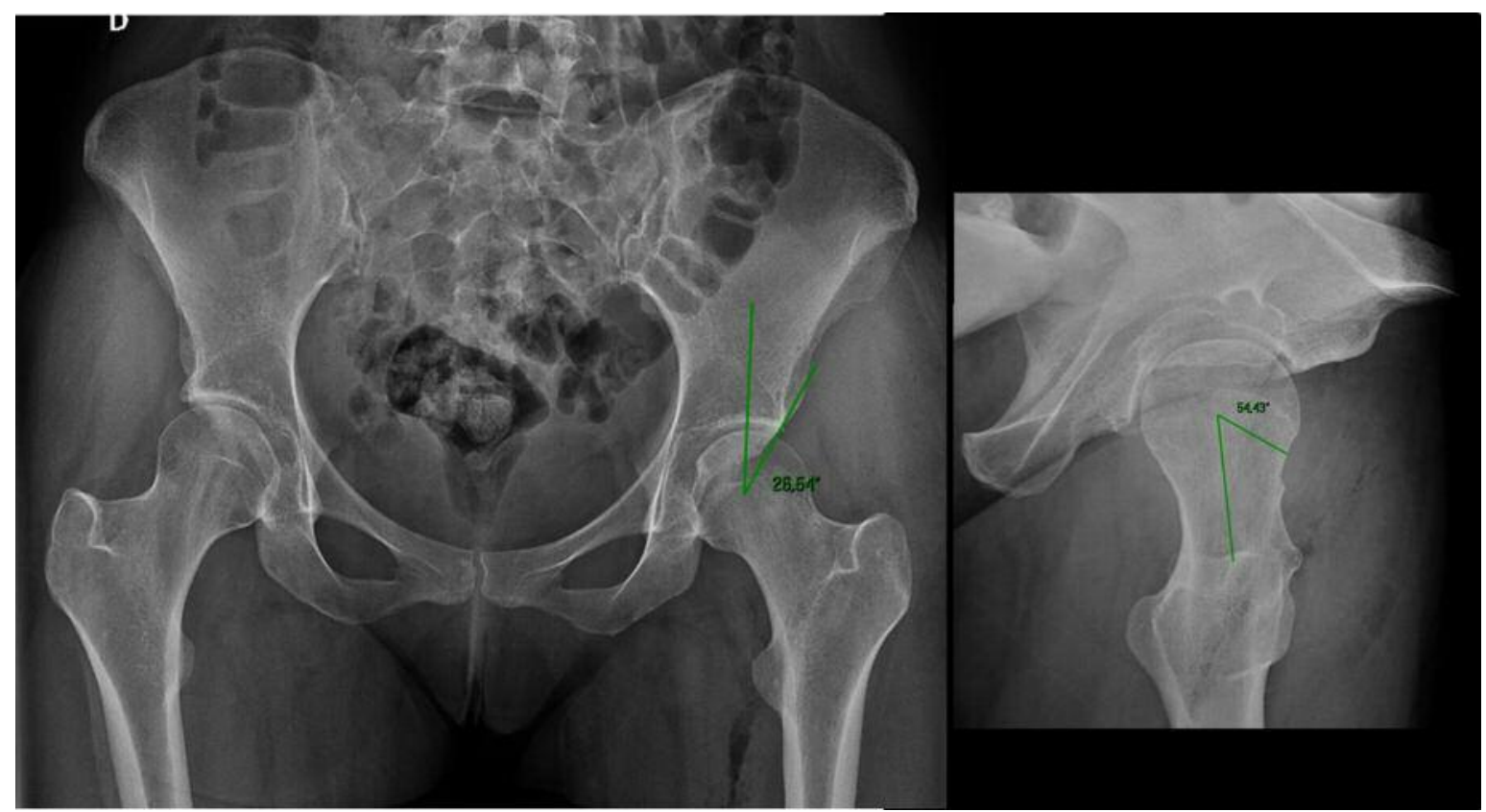

Figure 3. Postoperative imaging of the same patient.

Based on preoperative plain radiographs, 3 hips $(17 \%)$ were classified as having CAM-type impingement, 2 (12.5\%) were classified as PINCER-type, and 12 hips (70.5\%) as mixed. Ten hips (59\%) showed no osteoarthritis on X-ray (Tönnis grade 0 ) and 7 (41\%) showed signs of early osteoarthritis (grade 1 ). The median alpha angle decreased from $60^{\circ}$ to 46.5 , the median Weber angle from $34^{\circ}$ to $33.5^{\circ}$ and the femoral head-neck offset changed from $5.37 \mathrm{~mm}$ to 8 $\mathrm{mm}$ after the femoroplasty.

The median preoperative mHHS was 74.7 (range 39.6-93.4; $S D=17$ ), and the median preoperative VAIL score was 53 (range 30-78; $S D=15.1$ ). At final follow-up, the median mHHS increased to 95.8 (range 69-100; SD 8.4) and the median of VAIL score was 85 (range 57-100; DS=14.4). The differences were statistically significant for both scores $(p<0.001$; $p=0.026$ respectively). According to VAS score, a statistical significant improvement was also found from the preoperative to final follow-up, from a median of 5.5 (range 3-7) to 0 (range 0-5). There were no cases of worsening of osteoarthritis according Tönnis scale. Two patients referred a recurrence of pain one year after surgery who were treated conservatively. No other complications have been reported in this study.

\section{Discussion}

FAl is a recognized cause of hip pain, and arthroscopic treatment is gaining popularity worldwide. Patients undergoing hip arthroscopy for FAI and labral injuries showed excellent short-term results, significant increased clinical outcomes, high return-to-sport rates and few complications ${ }^{17,18}$. Due to the complex anatomic relationship between the iliopsoas tendon and the anterior surface of the hip joint, intra-articular pathologies can be associated with a iliopsoas tendinopathy and vice versa. Patients affected by internal snapping hip syndrome may show associated intra-articular lesions in more than $50 \%$ of cases $^{19}$. The iliopsoas tendon itself may be the cause of a labral injury. Domb et al. described an anterior labral injury at the 3 o'clock position directly beneath the iliopsoas tendon, which lies in an extra-articular position immediately beyond the joint capsule, which is the direct consequence of an iliopsoas impingement ${ }^{5}$. With the increasing number of primary hip arthroscopies being performed, surgeons are increasingly facing with patients with recurrent or persistent pain, and with the need of a revision surgery. Recent studies reported a revision rate after hip arthroscopy from 1.9 to $7.7 \%$, with a mean interval between the first arthroscopy and revision surgery of 25 months ${ }^{20,21}$. The most common cause for revision hip arthroscopy are residual $\mathrm{FAI}$ (from $68 \%$ to $81 \%$ of cases), labral pathology or reruptures (53\%), cartilage injuries $(36 \%)$, adhesions $(24 \%)$, ligamentum teres pathology $(15 \%)$, iliopsoas tendon pathology (13\%) and loose bodies $(6 \%)^{21,22}$. Gupta et al. recently reported that in $20 \%$ of patients who had a revision hip arthroscopy surgery an internal snapping hip was objectivated at the preoperative physical examination, and that an iliopsoas tendon release was performed in $37 \%$ of all patients ${ }^{23}$. A recent systematic review of the literature reported a mean incidence of psoas release during hip arthroscopy revision of $15 \%{ }^{24}$. It is now clear that an iliopsoas tendinopathy may influence long term results and that it can be the reason of revision surgery in an high percentage of cases. For this reason an 
accurate preoperative clinical examination is mandatory. Iliopsoas tendon pain may simulate a labral injury. Pain is located at the groin and is esacerbated with sports activities and with hip flexion below $90^{\circ}$. At the clinical examination the pain can be evocated by direct palpation of the iliopsoas tendon and/or by the isometric contraction of the hip flexors and the knee in moderate flexion.

Both open and endoscopic surgical techniques have been described for iliopsoas tendon release. Many articles have been reported in the literature about open surgical release or lengthening of the iliopsoas tendon. However the result of open surgery are moderate probably because of the impossibility to treat intra-articular pathologies. Furthermore, a complication's rate up to $40 \%$ of cases has been reported ${ }^{19}$. Encouraging results have been showed with endoscopic release. Higher success and less recurrence rate, fewer complications, and decreased postoperative pain compared to open surgery have been reported. Endoscopic iliopsoas release may be performed at the level of the lesser trochanter by and additional portal ${ }^{25}$, or by a transcapsular approach. In this case, the tendon release is performed across the hip joint through an anterior capsulotomy at the level of the labrum or at the femoral neck. Cadaveric studies showed some differences in performing tendon release at the lesser trochanter compared at the labrum ${ }^{26}$. At the level of the labrum, the muscle-tendineous junction consists of $60 \%$ muscle and $40 \%$ tendon. At the femoral neck it is about a $50-50 \%$, while at its insertion on the lesser trochanter, it consists of $40 \%$ muscle and $60 \%$ tendon. So, some Authors suggest that transcapsular release should be the ideal site for tenotomy because the muscular portion of the iliopsoas muscle is preserved, while releasing the tendon at the lesser trochanter would be equivalent to releasing the entire iliopsoas muscle belly-tendon complex ${ }^{27}$. For this reason we routinely perform transcapsular release of the iliopsoas tendon at the acetabular labrum. However, these conclusions are bases a cadaveric anatomic studies, and a comparative study showed no significant differences in clinical scores at long term follow-up between the two techniques $^{28}$.

Good to excellent results have been reported after endoscopic released of iliopsoas tendon for internal snapping hip syndrome, but we are not aware of studies reporting results of tendon release in arthroscopic treatment of FAl. Our study showed good to excellent clinical results, improved pain and a statistical significant improvement compared to preoperative scores, which can be compared to that of patients without a iliopsoas pathology published in literature. However, two patients referred persistent mild to moderate pain one year after surgery, which was managed conservatively. We evaluate all patients for clinical signs of iliopsoas tendinopathy preoperatively, and when they are positive, we routinely perform the tenotomy together with labral repair and others intraarticular procedures. A few things are important to keep in mind while performing iliopsoas release. Re- cent studies showed that a multiple iliopsoas tendon may exists in a large number of patients ${ }^{6,29}$. Philippon et al. ${ }^{9}$ in a cadaveric study found that the iliopsoas tendon was composed by two tendons in $64.2 \%$ of cadavers, by three tendons in $7.5 \%$ of cases and a single tendon in only in $28.3 \%$ of cases. These studies suggest that, once thought to be a rare anatomic variant, the finding of 2 or multiple iliopsoas tendons is very common, and that an incomplete release may be the cause of recurrence of groin pain and may require a revision surgery. So, if a tendon smaller than $10 \mathrm{~mm}$ of diameter is encountered, an additional tendon should be sought out, because it is probable that this represents only one portion of the tendon.

A significant loss of flexion strength is experienced by all patients after surgery. In a recent MRI study, atrophy of the iliacus muscle and of the psoas muscle were found in the $85 \%$ and $75 \%$ of patients respectively ${ }^{30}$. Even if atrophy was not associated with a significant decrease in hip function, and no significant differences in clinical outcomes were found at longterm follow-up between the operated hip and the contralateral healthy limb, patients should be informed of the loss of flexion strength after surgery, which normally improves from 6 to 10 months ${ }^{6,31}$. Finally, caution should be paid in performing iliopsoas tendon release in presence of risk factors of instability. The iliopsoas tendon is an important active stabilizer of the hip joint, and hip dysplasia, excessive acetabuloplasty, excessive capsulotomy, ligamentum teres resection, ligamentous laxity, and long distraction time resulting in ligament elongation, have been all recognized as potential risk factors for post-operative hip instability ${ }^{32}$. Micro-instability, early joint degeneration, subluxation and hip dislocation have been reported after labral repair, acetabular rim trimming and iliopsoas tenotomy, in particular in patients with hip dysplasia $^{33}$. Therefore, a careful selection of the patients avoid excessive acetabular rim trimming and capsulotomy are recommended in case of risk factors of hip instability.

This study has several limitation. One of the most important is the lack of control group consisting of patients treated with hip arthroscopy but without iliopsoas tendon release. Than, the relatively small sample size is a limitation of the study, making statistical analysis of the data difficult. Evaluation of outcome after hip arthroscopy is also challenged by the lack of dedicated subjective outcome instruments for patients with non-arthritic hip pathology. The loss of flexion strength was not measured. However this does not seem to affect the clinical score at long-term follow-up.

\section{Conclusion}

Iliopsoas tendinopathy can be associated to FAI in a large number of patients, and failure to diagnose and treat it may be the reason of poor results and a revision surgery. Arthroscopic iliopsoas tendon release seems to produce good clinical outcome in these patient, re- 
Arthroscopic release of iliopsoas tendon in patients with femoro-acetabular impingement: clinical results at mid-term follow-up

duce pain and the rate of a revision hip arthroscopy. Further studies involving more patients with longer follow-up are needed in order to evaluate the effectiveness of the treatment and the long-term outcomes.

\section{References}

1. Burman MS. Arthroscopy or the direct visualization of joints: an experimental cadaver study. 1931. Clin Orthop Relat Res. 2001;390:5-9.

2. Suzuki S, Awaya G, Okada Y, Maekawa M, Ikeda T, Tada H. Arthroscopic diagnosis of ruptured acetabular labrum. Acta Orthop Scand. 1986;57:513-515.

3. Kelly BT, Williams RJ 3rd, Philippon MJ. Hip arthroscopy: current indications, treatment options, and management issues. Am J Sports Med. 2003 Nov-Dec;31(6):1020-37.

4. Byrd JW, Jones KS. Hip arthroscopy in athletes: 10-year follow-up. Am J Sports Med. 2009;37:2140-3.

5. Domb BJ, Shindle MK, McArthur B, Voos JE, Magennis EM, Kelly BT. Iliopsoas impingement: a newly identified cause of labral pathology in the hip. HSS J. 2005;7:145-150.

6. Ilizaliturri VM, Suarez-Ahedo C, Acuña M. Internal Snapping Hip Syndrome: Incidence of Multiple-Tendon Existence and Outcome After Endoscopic Transcapsular Release. Arthroscopy. 2015;31:1991-5.

7. Khan M, Adamich J, Simunovic N, Philippon MJ, Bhandari M, Ayeni OR. Surgical management of internal snapping hip syndrome: a systematic review evaluating open and arthroscopic approaches. Arthroscopy. 2013;29:942-8.

8. Lewis CL. Extra-articular Snapping Hip:A Literature Review. Sports health. 2010;2:186-190.

9. Philippon MJ, Devitt BM, Campbell KJ et al. Anatomic Variance of the lliopsoas Tendon. Am J Sports Med. 2014 42: 807.

10. Padulo J, Oliva F, Frizziero A, Maffulli N. Muscles, Ligaments and Tendons Journal. Basic principles and recommendations in clinical and field science research: 2016 Update. MLTJ. 2016;6(1):1-5.

11. Byrd JW. Hip arthroscopy: the supine position. Clin Sports Med. 2001;20:703-731.

12. Cefin Barton C, Salineros MJ, Rakhra KS, Beaule PE. Validity of the Alpha Angle Measurement on Plain Radiographs in the Evaluation of Cam-type Femoroacetabular Impingement. Clin Orthop Relat Res. 2011;469:464-469.

13. Nemtala F, Mardones R, Tomic A. Anterior and Posterior Femoral Head-Neck Offset Ratio in the Cam Impingement. Cartilage. 2010;1:238-241.

14. Tannast M, Siebenrock KA, Anderson SE. Femoroacetabular Impingement: Radiographic Diagnosis-What the Radiologist Should Know. AJR 2007;188:1540-1552.

15. Terjesen T, Gunderson RB. Radiographic evaluation of osteoarthritis of the hip. An inter-observer study of 61 hips treated for late-detected developmental hip dislocation. Acta Orthop. 2012;83:185-189.

16. Byrd T. Modifed Anterior Portal for Hip Arthroscopy. Arthroscopy Tech. 2013:2:337-339.

17. Gupta A, Redmond JM, Stake CE, Dunne KE, Domb BG. Primary hip arthroscopy result in improved clinical outcomes? 2year clinical follow-up on a mixed group of 738 consecutive primary hip arthroscopies performed at a high-volume referral center. Am J Sports Med. 2015;28.

18. Torsten Grønbech Nielsen TG, Miller LL, Lund B, Christiansen $\mathrm{SE}$, Lind M. Outcome of arthroscopic treatment for symptomatic femoroacetabular impingement. BMC Musculoskeletal Disorders. 2014;15:394.

19. Hoskins JS, Burd TA, Allen WC. Surgical correction of internal coxa saltans: a 20-year consecutive study. Am J Sports Med. 2004.

20. Harris JD, McCormick FM, Abrams GD, et al. Complications and reoperations during and after hip arthroscopy: A systematic review of 92 studies and more than 6,000 patients. Arthroscopy. 2013;29:589-595.

21. Cvetanovich GL, Harris JD, M.D., Brandon J. Erickson BJ, Bach BR Jr., Charles A. Bush-Joseph CA, Nho SJ. Revision Hip Arthroscopy: A Systematic Review of Diagnoses, Operative Findings, and Outcomes. Arthroscopy. 2015.

22. Ljiljana Bogunovic MD, Meghan Gottlieb MSW, Gail Pashos BS, Geneva Baca BA, John C. Clohisy. Why Do Hip Arthroscopy Procedures Fail? Clin Orthop Relat Res. 2013; 471:2523-2529.

23. Gupta A, Redmond JM, Stake CE, Dunne KF, Hammarstedt $\mathrm{JE}$, Domb BG. Outcomes of Revision Hip Arthroscopy: 2-Year Clinical Follow-up. Arthroscopy. 2016.

24. Sardana V, Philippon MJ, de Sa D, Bedi A, Ye L, Simunovic N, Ayeni OR. Revision Hip Arthroscopy Indications and Outcomes: A Systematic Review. Arthroscopy. 2015.

25. Byrd JW. Evaluation and management of the snapping iliopsoas tendon. Tech Orthop. 2005:20:45-51.

26. Alpert JM, Kozanek M, Li G, Kelly BT, Asnis PD. Cross-sectional analysis of the iliopsoas tendon and its relationship to the acetabular labrum: an anatomic study. Am J Sports Med. 2009;37:1594-1598.

27. Blomberg JR, Zellner BS, Keene JS. Cross-sectional analysis of iliopsoas muscle-tendon units at the sites of arthroscopic tenotomies: an anatomic study. Am J Sports Med. 2011;39 Suppl:58S-63S.

28. Ilizaliturri VM, Buganza-Tepole M, Olivos-Meza A, Acuna M, Acosta-Rodriguez E. Central Compartment Release Versus Lesser Trochanter Release of the lliopsoas Tendon for the Treatment of Internal Snapping Hip: A Comparative Study. Arthroscopy. 2014;30:790-5.

29. Crompton T, Lloyd C, Kokkinakis M, Norman-Taylor F. The prevalence of bifid iliopsoas tendon on MRI in children. J Child Orthop. 2014;8:333-336.

30. Hain KS, Blankenbaker DG, De Smet AA, Keene JS, Munoz del Rio A. MR Appearance and Clinical Significance of Changes in the Hip Muscles and Iliopsoas Tendon After Arthroscopic lliopsoas Tenotomy in Symptomatic Patients. HSSJ. 2013:9:236-241.

31. Duck-Soo Hwang, Jung-Mo Hwang, Pil-Sung Kim, et al. Arthroscopic Treatment of Symptomatic Internal Snapping Hip with Combined Pathologies. Clinics in Orthopedic Surgery 2015;7:158-163.

32. Sansone M, Ahldèn J , Sward L, Eriksson T, Karlsson J. Total dislocation of the hip joint after arthroscopy and ileopsoas tenotomy. Knee Surg Sports Traumatol Arthrosc. 2013;2 1:420-423.

33. Mei-Dan O, McConkey MO, Brick M Catastrophic failure of hip arthroscopy due to iatrogenic instability: can partial division of the ligamentum teres and iliofemoral ligament cause subluxation? Arthroscopy. 2012;28:440-445. 